\title{
Éclairages cartographiques sur l'élection présidentielle brésilienne de 2018
}

Hervé Théry

\section{(2) OpenEdition}

Journals

Édition électronique

URL : http://journals.openedition.org/ideas/5377

DOI : $10.4000 /$ ideas.5377

ISSN : 1950-5701

Éditeur

Institut des Amériques

\section{Référence électronique}

Hervé Théry, «Éclairages cartographiques sur l'élection présidentielle brésilienne de 2018 », IdeAs [En ligne], 13 | 2019, mis en ligne le 01 mars 2019, consulté le 22 avril 2019. URL : http://

journals.openedition.org/ideas/5377; DOI : 10.4000/ideas.5377

Ce document a été généré automatiquement le 22 avril 2019

\section{cc) (†)}

IdeAs - Idées d'Amériques est mis à disposition selon les termes de la licence Creative Commons Attribution - Pas d'Utilisation Commerciale - Pas de Modification 4.0 International. 


\title{
Éclairages cartographiques sur l'élection présidentielle brésilienne de 2018
}

\author{
Hervé Théry
}

1 Le 28 octobre 2018 le Brésil a choisi Jair Messias Bolsonaro (PSL), pour être son $38^{\mathrm{e}}$ Président de la République. Un mois plutôt il était donné dans tous les sondages comme battu par tous les autres candidats possibles. Cette élection a donc été une énorme surprise pour tous les observateurs, à l'étranger mais aussi au Brésil. Le nouveau président était jusque-là très peu connu, n'ayant été pendant vingt-sept ans qu'un obscur député fédéral, et n'avait émergé de l'anonymat que lors du vote sur la destitution de la présidente Dilma Rousseff, lorsqu'il avait dédié le sien à la mémoire de l'un des pires tortionnaires de la période de dictature militaire (1964-1985).

2 Cent jours après sa prise de fonction (le $1^{\mathrm{er}}$ janvier 2019), le ton est donné et les scandales se sont déjà multipliés. Mais il est encore trop tôt pour évaluer de manière tangible les résultats de son action. L'objectif de la présente contribution est plutôt d'éclairer le lecteur sur les raisons de cette élection. Pour ce faire, et de façon à être complémentaire par rapport aux autres articles de ce dossier, nous avons choisi d'utiliser la cartographie comme moyen d'éclairage des résultats de cette élection.

3 Les cartes qui représentent ces résultats seront mises en perspective par rapport à d'autres, construites par le traitement de données de diverses natures, obtenues à partir de sources officielles et non officielles, de variables et d'indicateurs traditionnels ou plus innovants.

\section{Les résultats}

\section{Fin d'un cycle}

4 Notons tout d'abord que si la victoire de Bolsonaro a été une surprise, on s'attendait du moins à une défaite de son principal opposant, Fernando Haddad - le représentant du 
parti des travailleurs (PT), parti qui a été au pouvoir pendant treize ans, de 2003 à 2016. Le pays avait manifesté clairement, de différentes manières, qu'il voulait tourner cette page.

$5 \quad$ Le résultat marque donc la fin d'un cycle qui est bien décrit par une série de cartes (figure 1) montrant quel candidat est arrivé en tête dans chaque État au premier tour des élections présidentielles tenues de 1998 à 2018. Elles retracent la conquête progressive du pays par le PT entre 1998 (où il ne l'avait emporté que dans un seul État), jusqu'en 2002 (où il avait gagné dans tous sauf deux), puis son reflux de 2006 à 2018, où il n'a gardé de position dominante que dans un État amazonien et surtout dans le Nordeste qui lui est resté fidèle (à l'exception du Ceará ${ }^{1}$, fief de Ciro Gomes, arrivé troisième).

Figure 1 : Historique des élections et résultats du premier tour de l'élection présidentielle.

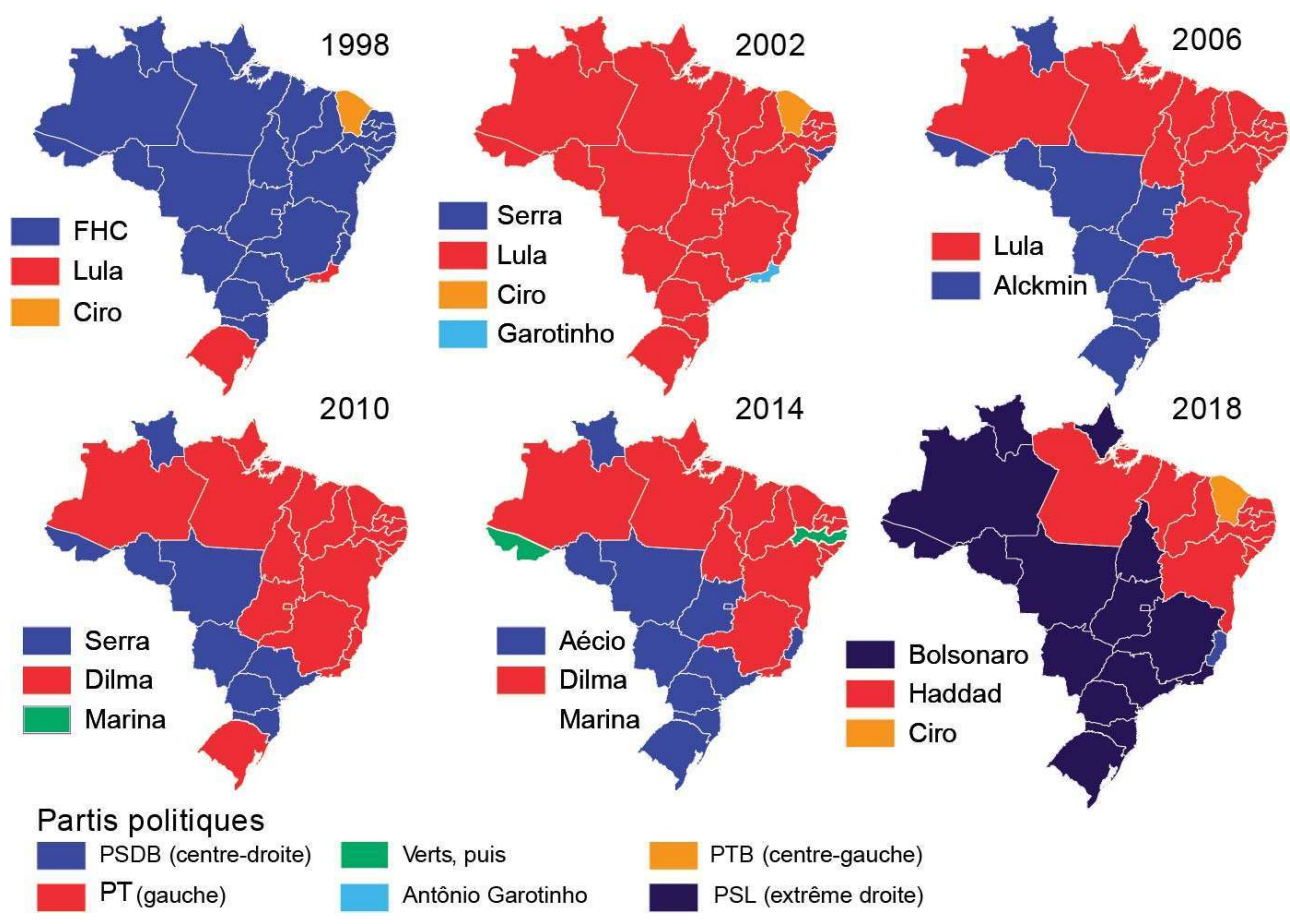

(c) HT/AN 2018

6 La carte des résultats de ce premier tour montre une très claire opposition entre deux ensembles spatiaux où chacun des deux candidats principaux est arrivé en tête, Haddad dominant l'essentiel du Nordeste (sauf le Ceará) et Jair Bolsonaro le Sudeste, le Sud et le Centre-Ouest. 
Figure 2 : Les résultats du premier tour de l'élection présidentielle de 2018.

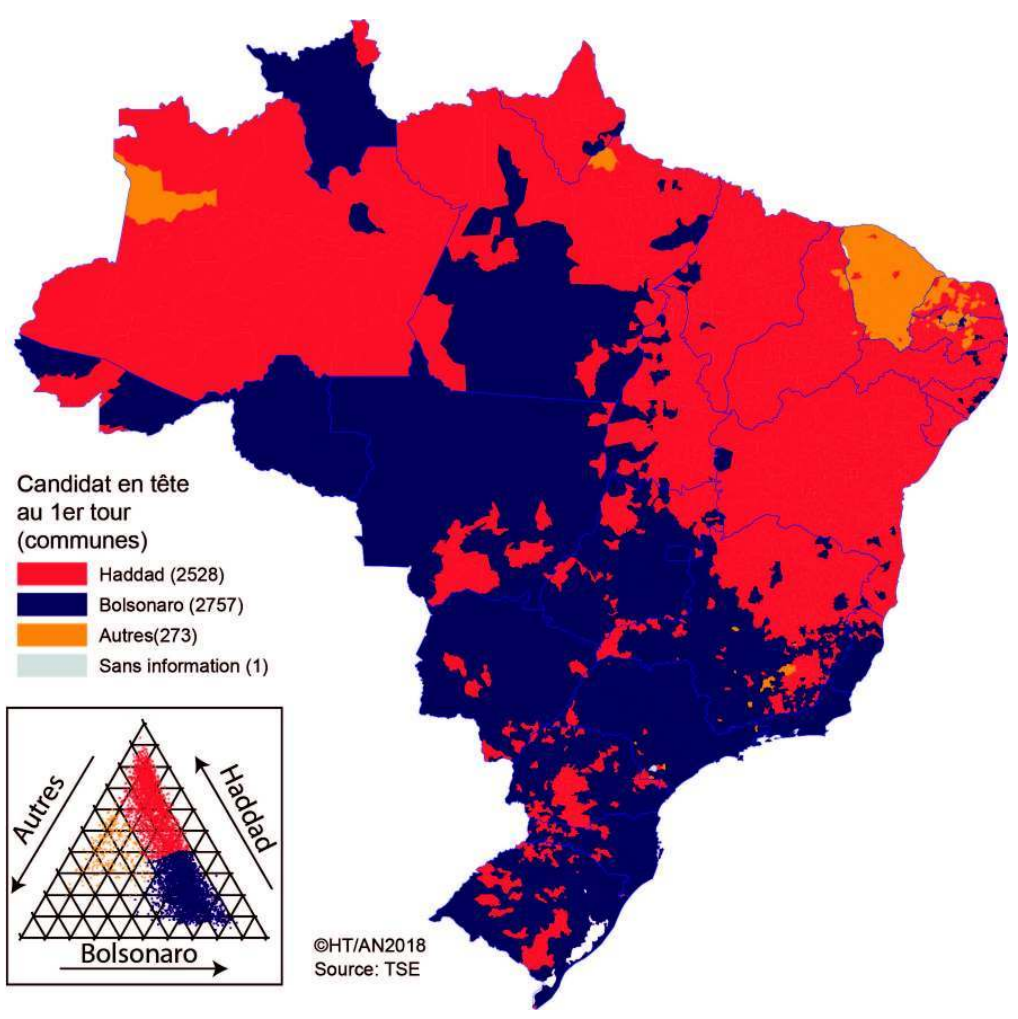

(c) HT/AN 2018

7 Au second tour, qui se jouait entre les deux candidats arrivés en tête au premier, Bolsonaro a remporté une nette victoire avec 57797847 voix (55,13\% des suffrages exprimés) contre 47040906 (44,87\%) à Fernando Haddad. Le nombre de communes où l'un ou l'autre a reçu le plus de votes est voisin, Haddad dans 2810 communes (sur les 5570 que compte le pays), et Bolsonaro dans 2760 , mais l'opposition géographique a été plus nette encore, Bolsonaro l'emportant au total pour avoir gagné dans les régions les plus peuplées du pays (figure 3).

Figure 3 : Les résultats du second tour.

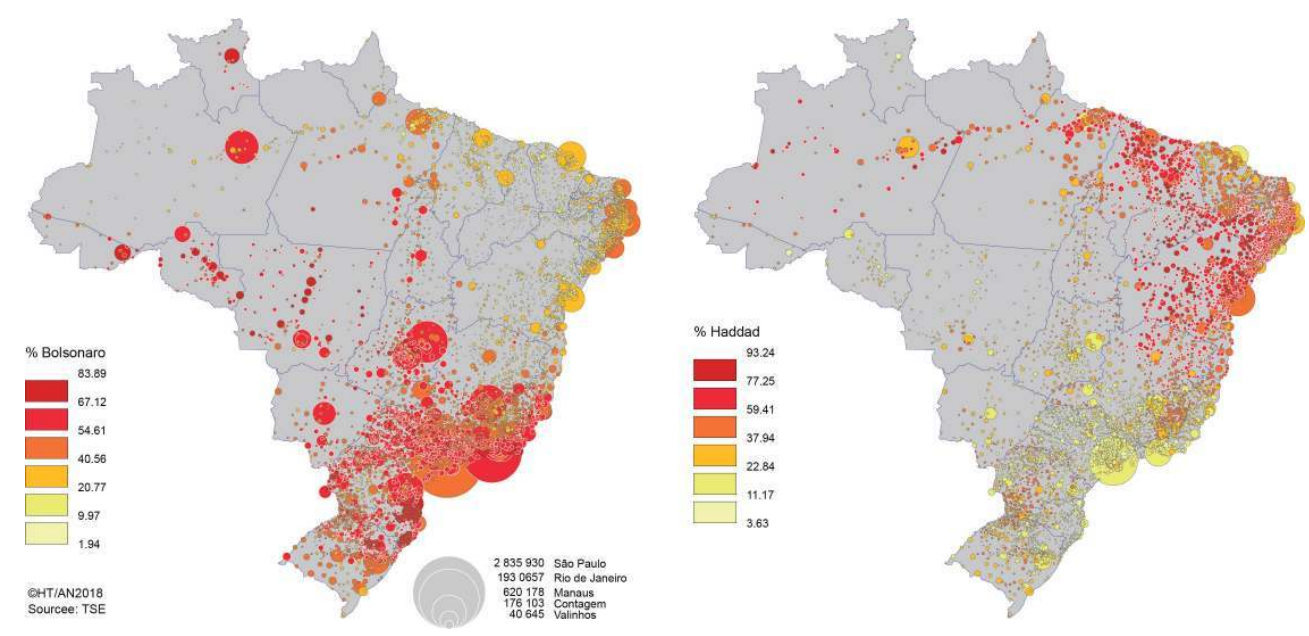

(c) HT/AN 2018 
Il y a donc une évidente composante géographique à la répartition des votes, en même temps qu'une composante sociale, et c'est ce que d'autres cartes peuvent aider à comprendre.

9 Une première explication est à chercher dans le fort contraste entre les niveaux de développement du Nord-Nordeste et du Sud-Sudeste, mais aussi avec du Centre-Ouest qui s'est beaucoup développé depuis les années 1980. Il est si fort qu'il est présent dans la plupart des cartes de l'Atlas do Brasil récemment publié (Théry H., et Mello-Théry N.A. de, 2018) l'on peut même dans beaucoup de cas parler de deux Brésils, l'un déjà «émergé " (Théry H., 2016) et l'autre encore marqué par la pauvreté.

\section{Deux Brésils}

10 La figure 4, qui fait la synthèse d'une série d'indicateurs socio-économiques, montre une ressemblance frappante avec les résultats de l'élection en opposant d'un côté l'essentiel du Nordeste et la plus grande partie de l'Amazonie, et de l'autre le Centre-Sud plus développé, auquel se sont ajoutés depuis une trentaine d'années le Centre-Ouest et une partie de l'Amazonie, aux confins du Pará, de l'Amapá et du Roraima.

Figure 4 : Synthèse d'indicateurs sociaux.

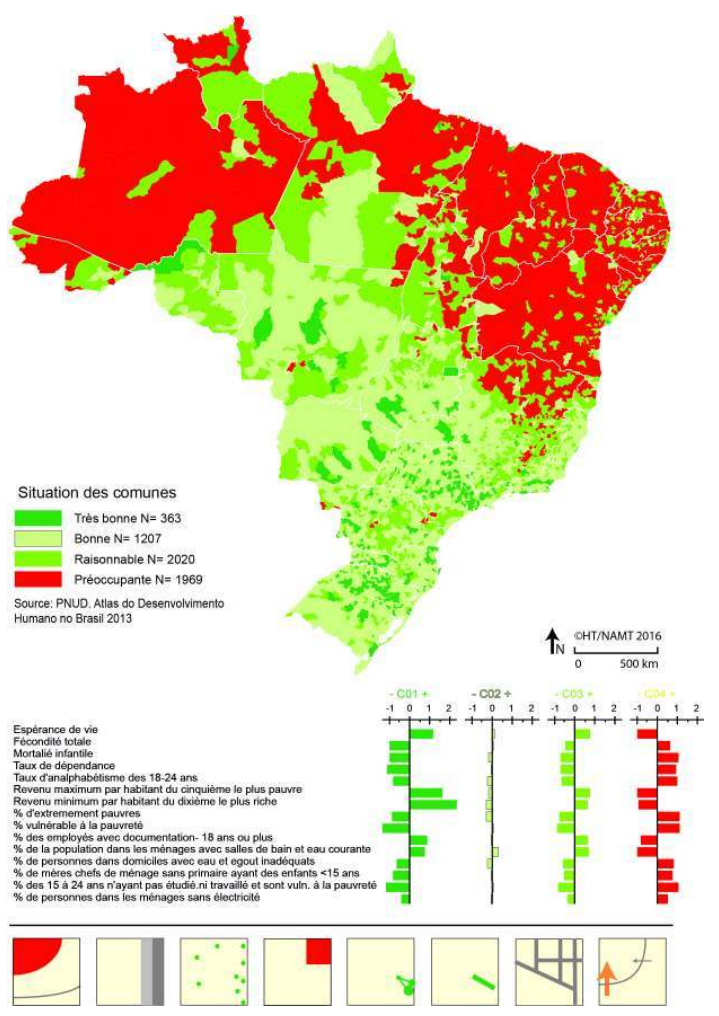

(c) HT/NAMT 2016

11 Un deuxième facteur, évidemment corrélé au premier, est que les régions les plus pauvres, et tout particulièrement le Nordeste, ont été les principales bénéficiaires des politiques sociales mises en place par les présidents " petistes », Luíz Inácio « Lula » da Silva (2003-2010) et Dilma Rousseff (2011-2016). 
12 Le plus connu de ces programmes sociaux est la Bolsa Familia (Bourse famille), qui a bénéficié en 2015 à 13,8 millions de familles (soit environ 50 millions de personnes sur les 207 millions que comptait alors le pays), dont la moitié dans la région Nordeste, la région la plus pauvre du pays. C'était avant tout une politique de correction des inégalités de revenus, non une politique territoriale, mais elle a eu de claires répercussions régionales puisque la pauvreté, notamment rurale, est territorialement très concentrée.

13 Ces programmes de lutte contre la pauvreté avaient beaucoup fait pour la popularité de Lula, notamment dans le Nordeste, et lui avaient permis d'être réélu en 2006 et de faire élire Dilma Rousseff en 2010 et 2014. Mais cette fois il n'a pas pu prendre part à l'élection, ayant été condamné à douze ans de prison pour corruption, ce qui l'a rendu inéligible alors que les sondages d'opinion lui donnaient près de $40 \%$ des intentions de vote. Le transfert de voix vers Fernando Haddad n'a pas été suffisant pour le faire élire, mais il est clair que c'est dans le Nordeste que ce dernier a eu ses meilleurs résultats.

L'évolution du nombre de familles bénéficiaires entre 2006 et 2014 va dans le même sens : on note une nette augmentation dans toutes les régions du Nordeste, du Nord, du CentreOuest, et une nette diminution dans le Sud et le Sudeste (sauf dans le nord du Minas Gerais). L'évolution négative apparaît également dans certaines capitales du Nordeste, alors que le nombre de bénéficiaires augmente considérablement à Rio de Janeiro et São Paulo, sans pour autant inverser la tendance politique dans ces métropoles gigantesques.

Figure 5 : Bénéficiaires de la Bolsa Família.

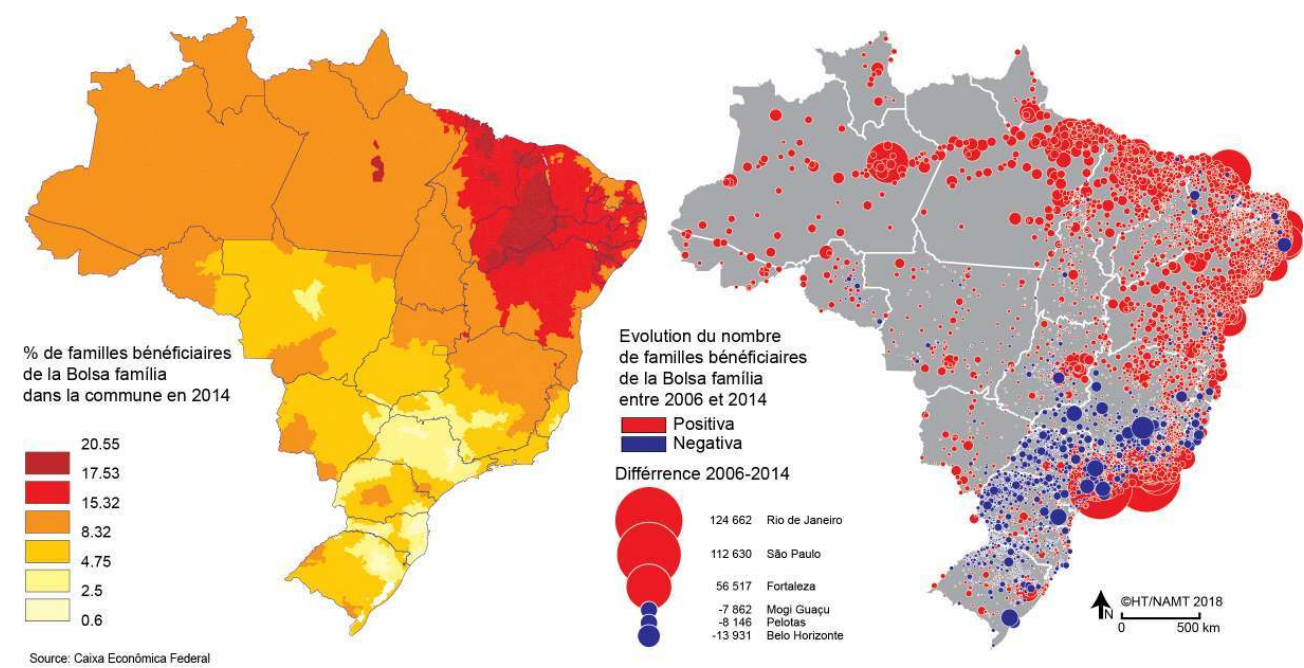

(C) HT/NAMT 2018

On a là l'une des clés du succès de Jair Bolsonaro : l'explication la plus plausible de son succès ne tient pas à son programme, puisqu'il n'en a pas vraiment, ni à la force de son parti, qu'il a rejoint récemment après avoir transité par neuf autres, ni un subit virage à droite d'un pays qui, lors des quatre dernières élections présidentielles, avait élu des présidents de gauche (deux fois Lula et deux fois Dilma Rousseff).

Moins qu'à une adhésion à ses idées, le succès de Bolsonaro a été dû pour l'essentiel à un rejet massif du PT dans une bonne partie de l'opinion, en dehors du Nordeste où les bénéficiaires des politiques sociales lui sont restés fidèles. Ailleurs les électeurs ont surtout retenu que son passage au pouvoir a été marqué par un reniement de ses promesses de réformes et un recours massif à la corruption. Ne pas engager les réformes 
structurelles qui auraient été nécessaires lui a aliéné le soutien de son aile gauche et la corruption lui a fait perdre le respect et le soutien de la classe moyenne, attachée aux valeurs d'honnêteté et de mérite que le parti mettait en avant quand il était dans l'opposition.

Une fois la candidature de Lula invalidée, la route était ouverte pour Jair Bolsonaro, qui a bénéficié de plusieurs facteurs favorables. D'une part, un groupe de généraux en retraite (dont le général Mourão, qui s'est engagé à ses côtés comme candidat à la viceprésidence) s'est employé à convaincre les généraux en activité que son élection serait acceptable. D'autre part, les milieux d'affaires ont abandonné leur candidat préféré, Geraldo Alckmin, dont la candidature n'a jamais décollé tant il a peu de charisme ${ }^{2}$ et rallié la sienne: dès le lendemain du premier tour, les cours de la Bourse ont commencé à monter, et le dollar - la valeur-refuge par excellence - a baissé de près de $3 \%$. Mais l'essentiel de son soutien est venu d'ailleurs.

\section{Les 3B}

Bolsonaro a en effet obtenu l'appui de trois groupes distincts, les protestants pentecôtistes, les éleveurs de bovins (ou plus généralement l'agrobusiness) et ceux qui prônent l'usage de la violence pour réduire l'insécurité. Il a fait ainsi, en sa faveur, l'alliance de ce qu'on appelle au Brésil les «trois B », «Bíblia, boi e bala » (Bible, bœuf et balle).

\section{Boi}

Ce n'est pas un hasard si on a symbolisé le groupe de pression qui représente les intérêts de l'agro-industrie par la figure du bœuf. Les éleveurs en constituent en effet l'un des segments les plus actifs, et celui qui a le plus activement appuyé la candidature de Bolsonaro. Il fait partie d'un vaste complexe agro-industriel, dit aussi "agro-business", fondé sur de grandes cultures tournées vers l'exportation comme la canne à sucre et le café (pour lesquelles le Brésil est au premier rang mondial), mais aussi plus récemment l'élevage bovin et la culture du soja, du maïs et du coton, qui ont conquis le Centre-Ouest depuis les années 1970.

Ce n'est toutefois qu'une partie du monde rural brésilien, et certaines régions, dans le Sud et surtout le Nordeste, sont occupées par la petite agriculture familiale, et l'on a pu dire que le Brésil avait deux agricultures distinctes. Cette affirmation est confirmée par le fait que, jusqu'à une date récente, le pays avait non pas un ministère de l'agriculture mais deux, l'un qui appuyait l'expansion du secteur agroindustriel tandis que l'autre, celui du Développement agraire, s'occupait de la petite agriculture familiale. C'est si vrai que dans le gouvernement de Michel Temer, qui a succédé à Dilma Rousseff après sa destitution, le deuxième ministère avait été rattaché à celui des affaires sociales.

21 L'opposition de ces deux mondes est bien illustrée par le contraste entre la prédominance des petites et très petites exploitations agricoles dans le Nordeste et des très grandes dans le Centre-Ouest (figure 6). 
Figure 6 : Latifundios et minifundios.

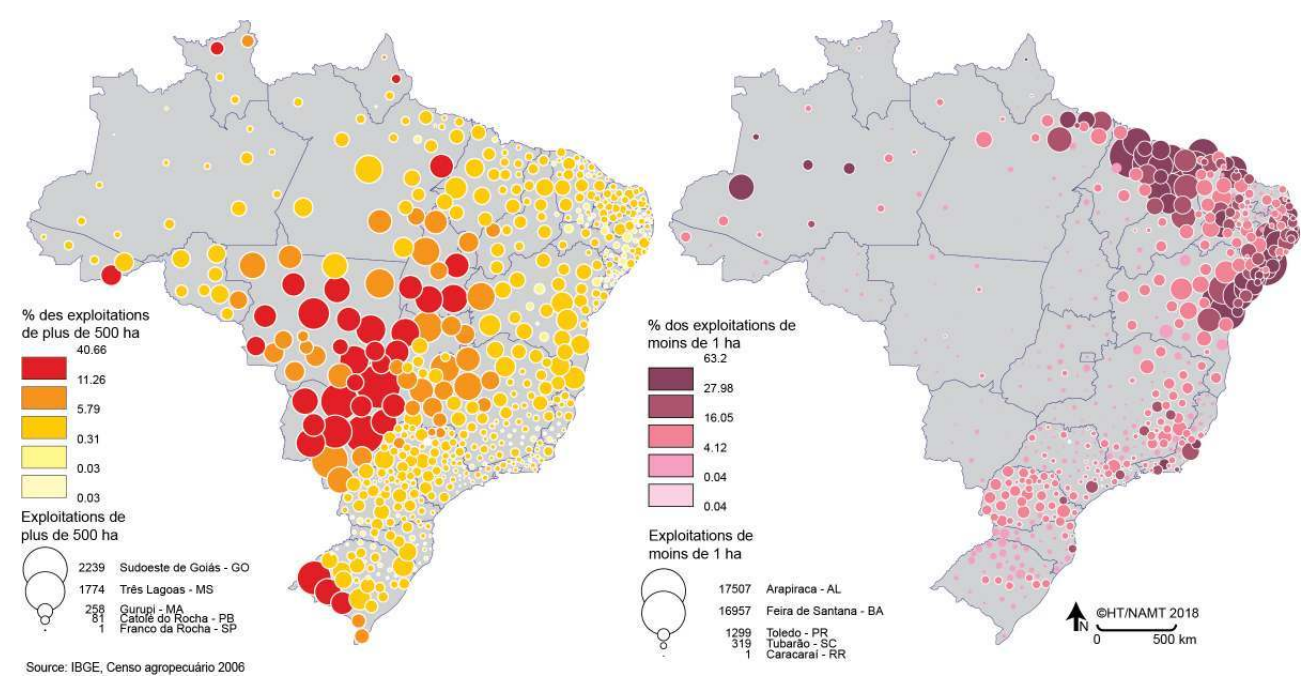

(C) HT/NAMT 2018

Or ces très grandes exploitations sont en effet le plus souvent consacrées à l'élevage bovin, au point que dans beaucoup de régions le nombre de têtes de bétail est très supérieur à celui des humains. Et si, à l'échelle nationale, le nombre de têtes de bétail est déjà un peu supérieur à celui des habitants, la figure 7 montre que dans le Centre-Ouest, qui a voté massivement pour Bolsonaro, il l'est bien davantage, jusqu'à quarante pour un. C'est l'un des fronts pionniers les plus actifs du pays, qui se déplace vers le nord et l'ouest du pays, avec ce que cela implique de déboisement, de conflits fonciers et de violence. La figure 7 confirme la co-incidence (qui n'est pas une coïncidence) entre la progression du front pionnier et le vote en faveur de Bolsonaro dans le Centre-Ouest, une sorte de Middle West brésilien mais avec bien des traits de Far West. 
Figure 7 : Humains et bovins.

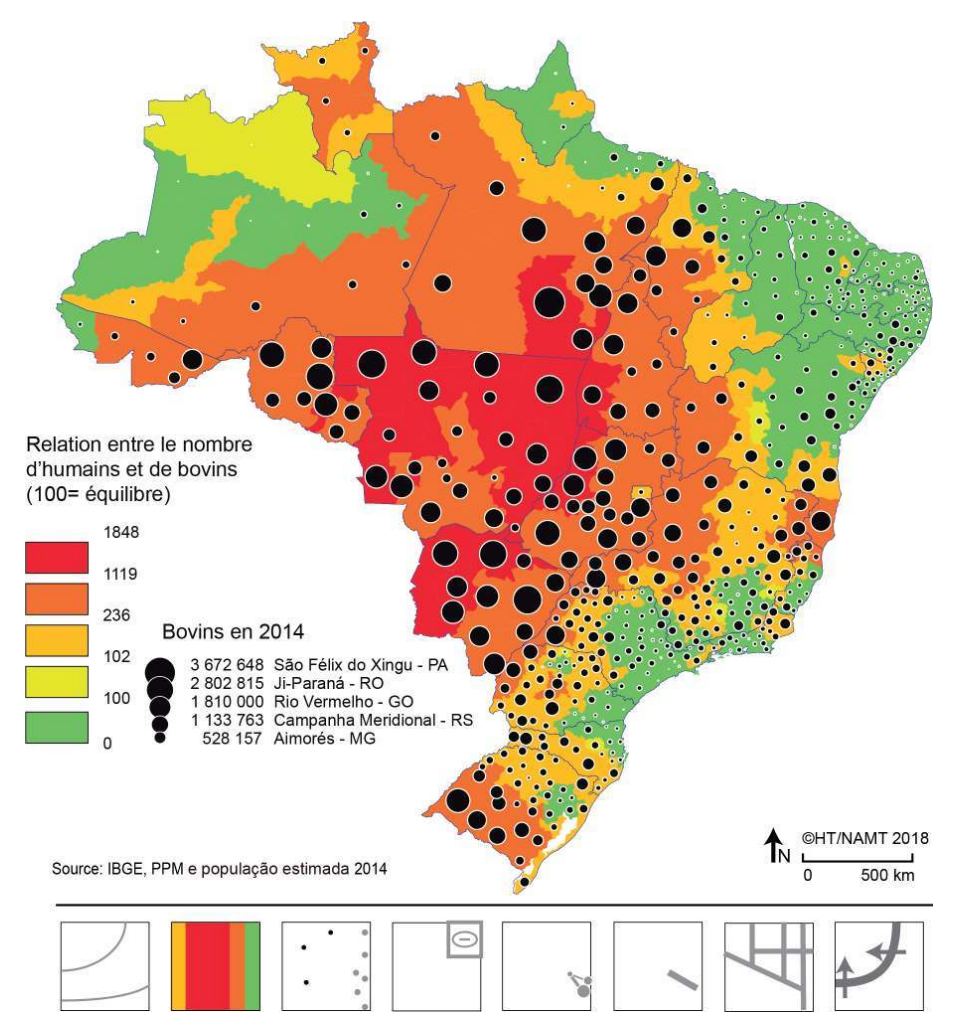

(c) HT/NAMT 2018

\section{Bíblia}

Le deuxième « $\mathrm{B}$ ", pour Bíblia (Bible) représente la puissance croissante des protestants pentecôtistes, qui ont fortement appuyé Jair Bolsonaro. Bien qu'il soit lui-même catholique pratiquant, Jair Bolsonaro a obtenu le ralliement de plusieurs de ces groupes « evangélicos ", dont l'influence s'accroît constamment dans le pays, au détriment de l'Église catholique (au rythme d'environ $1 \%$ par an). Il s'est même fait «baptiser » dans les eaux $\mathrm{du}$ Jourdain pour manifester encore plus clairement son rapprochement avec ce groupe dont les idées sont souvent proches des siennes ${ }^{3}$.

C'est une des clés de son succès, car leurs pasteurs ne se sont pas privés de donner des consignes de vote à leurs ouailles, qui suivent rigoureusement leurs consignes puisqu'une des raisons de l'avancée de ces nouvelles religions (qui ont par bien des aspects un comportement de sectes) est précisément que les fidèles y trouvent un encadrement religieux mais aussi moral et politique qui les rassure.

La dimension religieuse du vote est nette sur la figure 8 , où le Nordeste, le fief électoral de Fernando Haddad, est aussi le bastion de résistance du catholicisme, alors que les pentecôtistes sont particulièrement dynamiques dans le Centre-Ouest : l'esprit pionnier, le protestantisme et le capitalisme se combinent bien avec le conservatisme politique, une convergence bien connue depuis Max Weber et qui a encore été confirmée par l'élection de Donald Trump aux États-Unis... 
Figure 8 : Typologie des religions.

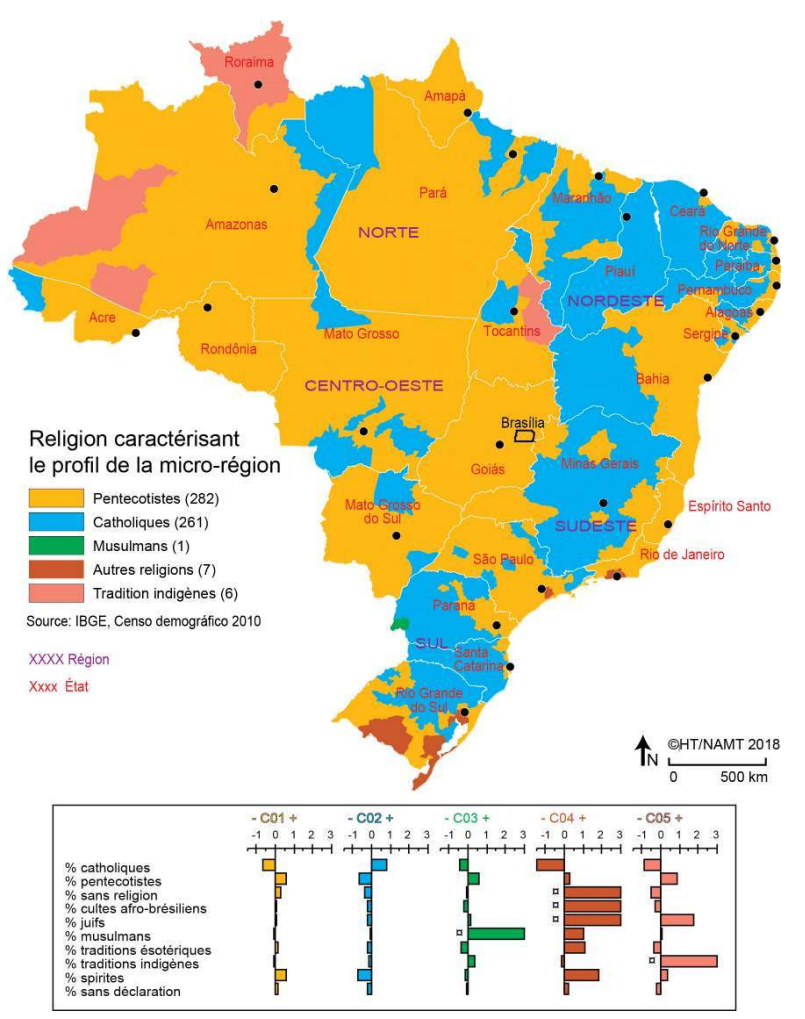

(c) HT/NAMT 2018

\section{Bala}

26 Le troisième B pour bala (balle) est celui qui a le plus attiré l'attention - et l'inquiétude des observateurs étrangers sur le nouveau président, qui a obtenu l'appui des groupes qui revendiquent une plus grande fermeté dans la répression de la criminalité.

Celle-ci est en effet préoccupante au Brésil, qui est l'un des pays les plus violents au monde, avec près de 60000 homicides par an. C'est un niveau comparable à celui de l'Inde, mais comme celle-ci, avec plus de 1,3 milliard d'habitants, est beaucoup plus peuplée que lui, le taux par habitant est évidemment bien plus élevé au Brésil. On notera toutefois - faible consolation - qu'il l'est moins que celui du Venezuela ou de l'Amérique centrale, qui détiennent en ce domaine un triste record mondial. 
Figure 9 : Nombre et taux d'homicides en 2015.

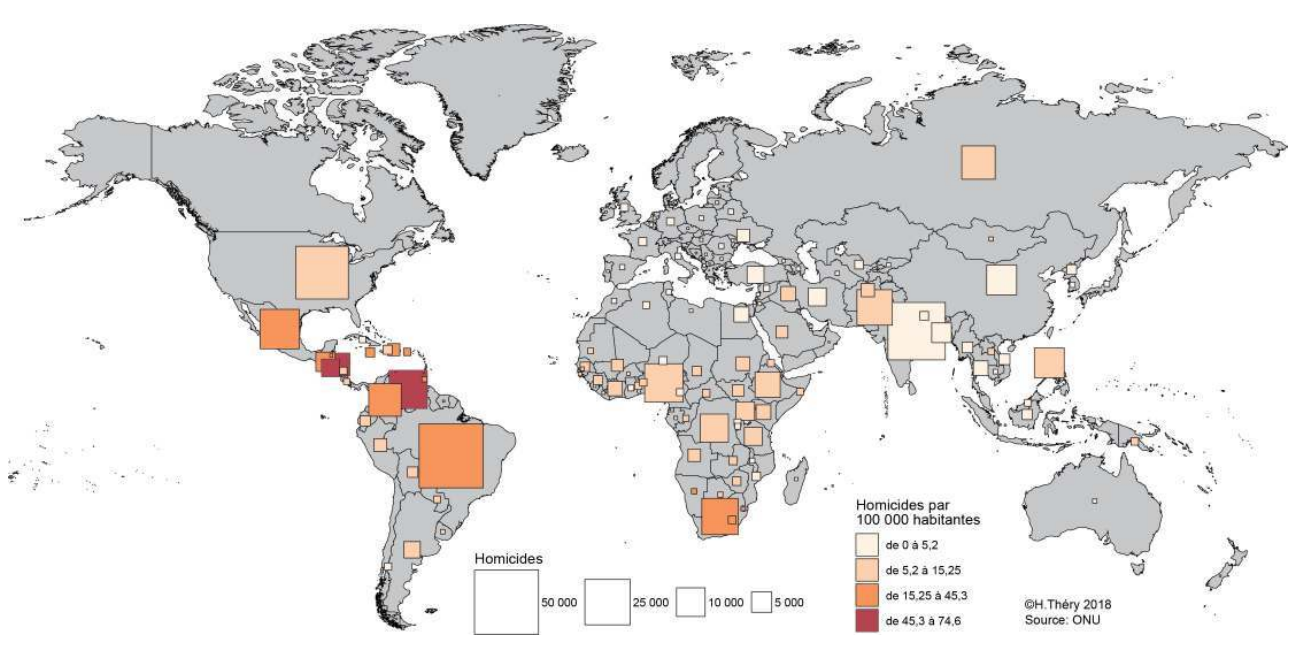

(C) Hervé Théry 2018

À l'intérieur du pays la criminalité qui attire le plus d'attention est celle des grandes villes, en particulier Rio de Janeiro, mais si l'on rapporte le nombre d'assassinats à la population, on s'aperçoit que c'est sur les fronts pionniers amazoniens que le nombre de morts par rapport à la population et la part des homicides dans les causes de mortalité sont les plus élevés, ce qui renforce le parallèle avec le Far West.

Figure 10 : Homicides et mortalité.
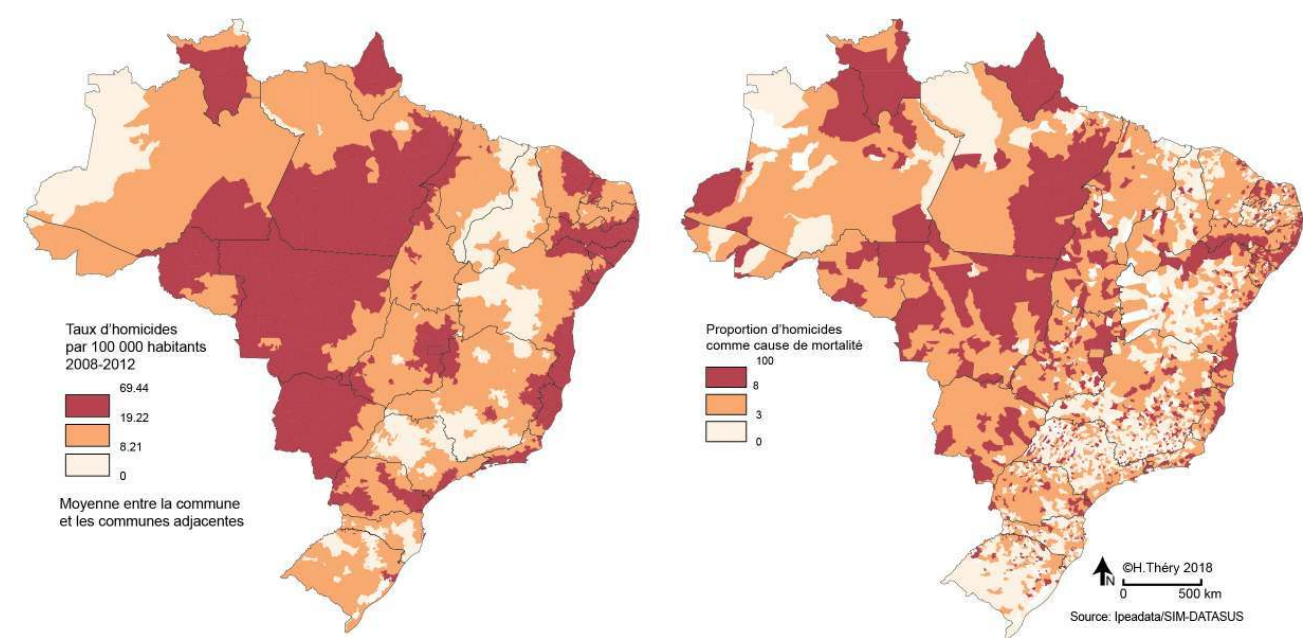

(c) Hervé Théry 2018

En insistant sur ce point, Jair Bolsonaro a eu le talent, comme c'est fréquemment le cas des leaders populistes, de sentir quels étaient les problèmes qui préoccupent réellement la population, même s'ils y apportent en général de mauvaises réponses. La solution qu'il a proposée - très probablement inadaptée - a été de permettre aux citoyens de s'armer et l'un de ses premiers actes a été de passer, dès son arrivée au pouvoir, un décret-loi autorisant les citoyens à posséder chacun jusqu'à quatre armes.

Cela n'a pas surpris les Brésiliens, puisque c'était une promesse de campagne. Reste à savoir s'il s'agissait de flatter les secteurs les plus extrêmes de son électorat, ou de convictions réelles, et surtout s'il voudra - et pourra - fonder sur celles-ci son action et 
celle de son gouvernement. Aucun commentateur ne se risque à imaginer ce que sera celle-ci, mais l'on peut au moins prévoir, sans grand risque de se tromper, que ce sera une période difficile, car, comme on l'a vu, son élection résulte du ralliement de groupes sociaux très différents.

\section{BIBLIOGRAPHIE}

Théry, Hervé et Mello-Théry, Neli Aparecida de, Atlas do Brasil, Disparidades e dinâmicas do território , São Paulo, Edições da Universidade de São Paulo EDUSP, 2018 ( $3^{\mathrm{e}}$ édition).

Théry, Hervé, Le Brésil, pays émergé, Paris, Armand Colin, coll. « Perspectives géopolitiques », 2016.

\section{NOTES}

1. Voir le nom des États et régions sur la figure 8.

2. En témoigne le surnom dont il a été affublé, « sorvete de chuchu », que l'on pourrait transposer bien qu'il s'agisse d'un autre légume - en « sorbet de navet».

3. Voir l'article de Morgane Reina dans le présent dossier.

\section{AUTEUR}

\section{HERVÉ THÉRY}

Directeur de recherche émérite au Creda (UMR7227 CNRS-Université Paris 3 Sorbonne-Nouvelle), professeur à l'Universidade de São Paulo (PPGH/FFLCH e GPP/EACH), hthery@aol.com 